\title{
Selection of the number of clusters via the bootstrap
}

\author{
Yixin Fang* \\ Division of Biostatistics, Department of Environmental Medicine \\ New York University \\ Email: Yixin.Fang@nyumc.org \\ Junhui Wang \\ Department of Mathematics, Statistics, and Computer Science \\ University of Illinois at Chicago \\ Email: junhui@uic.edu
}

\begin{abstract}
Here the problem of selecting the number of clusters in cluster analysis is considered. Recently, the concept of clustering stability, which measures the robustness of any given clustering algorithm, has been utilized in Wang (2010) for selecting the number of clusters through cross validation. In this manuscript, an estimation scheme for clustering instability is developed based on the bootstrap, and then the number of clusters is selected so that the corresponding estimated clustering instability is minimized. The proposed selection criterion's effectiveness is demonstrated on simulations and real examples.
\end{abstract}

Keywords: Cluster analysis; K-means; Spectral clustering; Stability.

*Correspondence to: Yixin Fang, 650 First Avenue, Room 551, New York, NY 10016, U.S.A. 


\section{Introduction}

The goal of cluster analysis is to assign observations into clusters so that observations in 2 the same cluster are similar in some sense. Popular methods include k-means clustering 3 (MacQueen, 1967), hierarchical clustering (Johnson, 1967), k-medoids clustering (Kaufman 4 and Rousseeuw, 1990), and spectral clustering (Shi and Malik, 2000). Many selection criteria 5 have been proposed for selecting the number of clusters in a clustering algorithm. Most 6 of them are based on between-cluster and/or within-cluster sum of squared distances; to 7 name just a few, Calinski and Harabasz (1974), Hartigan (1975), and Krzanowski and Lai 8 (1985). Milligan and Cooper (1985) conducted comprehensive simulation studies to evaluate 9 the performances of 30 different such procedures. Additionally, other procedures such as the 10 silhouette statistic proposed by Kaufman and Rousseeuw (1990), the gap statistic proposed 11 by Tibshirani, Walther and Hastie (2001), and the jump statistic proposed by Sugar and 12 James (2003) can also be applied to select the number of clusters. 13

Recently, the concept of clustering stability has drawn great attention from statisticians. $\quad 14$ Steinley (2008) developed a procedure, called stability analysis in his paper, for selecting 15 the number of clusters by repeatedly performing k-means many times with different random $\quad 16$ initializations. Steinley (2008) also mentioned three other types of stability: Bryant (2002) 17 developed a method to determine the general stability of the data with respect to the choice 18 of hierarchical clustering methods; Cheng and Milligan (1996) and some other methods were 19 concerned with character stability when characters are added to or removed from the system; 20 and McIntyre and Blashfield (1980) defined stability as the ability of a cluster solution to be ${ }_{21}$ continually recognized in different random samples of the general population. 22

Here we are concerned with the fourth type of stability, the one measuring the clustering 23 robustness against the randomness in the sample. Also see Fowlkes and Mallows (1983), 24 Gnanadesikan (1997), Ben-Hur, Elisseeff, and Guyon (2002), Lange et al. (2004), Ben-David, 25 
von Luxburg, and Pal (2006), and the references therein. As discussed in Wang (2010), the 1 intuition is that if we repeatedly draw samples from the population and apply the given 2 clustering algorithm, a good one should produce clusterings that do not vary much from one 3 sample to another. The stability measure is assumption free and applicable to both distance 4 based and non-distance based clustering algorithms.

It has been proposed to select the number of clusters as the one maximizing the clus- 6 tering stability. Since maximizing the clustering stability is equivalent to minimizing the 7 clustering instability, an estimation scheme for the clustering instability based on modified 8 cross-validation was developed in Wang (2010). The key idea in Wang (2010) is to split the 9 data into two training datasets and one validation dataset, where the two training datasets are 10 used to construct two clusterings and the validation dataset is used to measure the clustering 11 instability. However, the data splitting reduces the sizes of training datasets and therefore 12 the cross-validation method is inefficient. 13

In this manuscript, we develop an estimation scheme for clustering instability based on 14 the bootstrap. For an introduction to the bootstrap, please refer to Efron and Tibshirani 15 (1993). The implementation of the bootstrap method is straightforward, and it has a number 16 of advantages. First, the bootstrap samples are of the same size as that of the original data, 17 and therefore the bootstrap method is more efficient. Second, the bootstrap estimate of the 18 clustering instability is the nonparametric maximum likelihood estimate (MLE). Third, the 19 bootstrap method can provide the instability path of a clustering algorithm for any given 20 number of clusters. The last advantage is discussed in detail in Subsection 2.3. 21

The rest of the manuscript is organized as follows. In Section 2, the concept of clustering 22 stability is introduced and the bootstrap method is developed. In Section 3, the effectiveness 23 of the proposed method is demonstrated on simulations and two real data examples. Some 24 discussion is presented in Section 4 . 25 


\section{The bootstrap method}

\subsection{The clustering instability}

This subsection is almost reproducing Section 2 in Wang (2010), but with different notation 3 and expressions. Assume that $X^{n}=\left\{x_{1}, \ldots, x_{n}\right\}$ is a random sample of size $n$ from some 4 unknown distribution $F(x)$ with $x \in \mathbb{R}^{p}$, where $p$ is the number of features. A clustering 5 $\psi(x)$ is defined as a mapping $\psi: \mathbb{R}^{p} \rightarrow\{1, \ldots, k\}$, where $k$ is the given number of clusters. A 6 clustering algorithm $\Psi(\cdot ; k)$ with a given number of clusters $k \geq 2$ yields a clustering mapping $\quad 7$ $\Psi_{X^{n}, k}(x)$ when applied to sample $X^{n}$. Here the case of $k=1$ is excluded as $\Psi(\cdot ; 1)$ leads to 8 the same degenerate clustering $\Psi_{X^{n}, 1}(x)=1$ regardless of $X^{n}$.

Definition 1 (Clustering distance). The distance between any two clustering $\psi_{1}(x)$ and 10 $\psi_{2}(x)$ is defined as

$$
d_{F}\left(\psi_{1}, \psi_{2}\right)=E_{x^{0} \sim F, y^{0} \sim F}\left\{\left|I\left\{\psi_{1}\left(x^{0}\right)=\psi_{1}\left(y^{0}\right)\right\}-I\left\{\psi_{2}\left(x^{0}\right)=\psi_{2}\left(y^{0}\right)\right\}\right|\right\},
$$

where $I\{\cdot\}$ is the indicator function, and the expectation is taken over $x^{0}$ and $y^{0}$, two inde- ${ }_{12}$ pendent observations sampled from $F$.

The above expectation equals $\operatorname{Prob}\left\{\psi_{1}\left(x^{0}\right)=\psi_{1}\left(y^{0}\right), \psi_{2}\left(x^{0}\right) \neq \psi_{2}\left(y^{0}\right)\right\}+\operatorname{Prob}\left\{\psi_{1}\left(x^{0}\right) \neq \quad{ }_{14}\right.$ $\left.\psi_{1}\left(y^{0}\right), \psi_{2}\left(x^{0}\right)=\psi_{2}\left(y^{0}\right)\right\}$, so this distance measures the disagreement between two clusterings. $\quad 15$

Definition 2 (Clustering instability). The clustering instability of $\Psi(\cdot ; k)$ is defined as

$$
s(\Psi, k, n)=E_{X^{n} \sim F^{n}, \tilde{X}^{n} \sim F^{n}}\left\{d_{F}\left(\Psi_{X^{n}, k}, \Psi_{\tilde{X}^{n}, k}\right)\right\}
$$

where the expectation is taken over $X^{n}$ and $\tilde{X}^{n}$, two independent random samples of size $n \quad 17$ from $F$, and $\Psi_{X^{n}, k}$ and $\Psi_{\tilde{X}^{n}, k}$ are two clusterings trained from $X^{n}$ and $\tilde{X}^{n}$ respectively. 18

By definition, small values of $s(\Psi, k, n)$ indicate a stable clustering algorithm $\Psi(\cdot ; k) . \quad 19$ Then the optimal number of clusters is defined as 20

$$
k_{0}=k_{0}(n)=\operatorname{argmin}_{2 \leq k \leq K} s(\Psi, k, n),
$$

where $K$ is preset as the maximum number of clusters to be considered in practice. 


\subsection{The bootstrap method for selecting the number of clusters}

Because the random sample $X^{n}$ is generated from $F$, denote $s(\Psi, k, n)$ as $\theta(F)$, a function $\quad 2$ of $F$. We wish to estimate $\theta(F)$ on the basis of $X^{n}$. Let $\hat{F}$ be the empirical distribution, 3 putting probability $1 / n$ on each of the observed values $x_{i}, i=1, \ldots, n$. We are interested in 4 using the plug-in estimator, $\theta(\hat{F})$, to estimate $\theta(F)$. Note that $\hat{F}$ is the nonparametric MLE 5 of $F$, and consequently $\theta(\hat{F})$ is the nonparametric MLE of $\theta(F)$. 6

Noting that $\theta(F)$ can be written as $\quad 7$ $E_{X^{n} \sim F^{n}, \tilde{X}^{n} \sim F^{n}} E_{x^{0} \sim F, y^{0} \sim F}\left\{\left|I\left\{\Psi_{X^{n}, k}\left(x^{0}\right)=\Psi_{X^{n}, k}\left(y^{0}\right)\right\}-I\left\{\Psi_{\tilde{X}^{n}, k}\left(x^{0}\right)=\Psi_{\tilde{X}^{n}, k}\left(y^{0}\right)\right\}\right|\right\}$,

its plug-in estimator $\theta(\hat{F})$ is equal to

$E_{X^{n *} \sim \hat{F}^{n}, \tilde{X}^{n *} \sim \hat{F}^{n}} E_{x^{0 *} \sim \hat{F}, y^{0 *} \sim \hat{F}}\left\{\left|I\left\{\Psi_{X^{n *}, k}\left(x^{0 *}\right)=\Psi_{X^{n *}, k}\left(y^{0 *}\right)\right\}-I\left\{\Psi_{\tilde{X}^{n *}, k}\left(x^{0 *}\right)=\Psi_{\tilde{X}^{n *}, k}\left(y^{0 *}\right)\right\}\right|\right\}$.

Since the inside expectation can be simplified, the plug-in estimator becomes

$E_{X^{n *} \sim \hat{F}^{n}, \tilde{X}^{n *} \sim \hat{F}^{n}}\left\{\frac{1}{n^{2}} \sum_{i=1}^{n} \sum_{j=1}^{n}\left|I\left\{\Psi_{X^{n *}, k}\left(x_{i}\right)=\Psi_{X^{n *}, k}\left(x_{j}\right)\right\}-I\left\{\Psi_{\tilde{X}^{n *}, k}\left(x_{i}\right)=\Psi_{\tilde{X}^{n *}, k}\left(x_{j}\right)\right\}\right|\right\}$.

Now it is ready to present the bootstrap method for estimating the clustering instability 10 $s(\Psi, k, n)$, as well as the optimal number of clusters $k_{0}$.

Algorithm 1: Bootstrap method for selecting the number of clusters

Step 1. Generate $B$ independent bootstrap sample-pairs $\left(X_{b}^{n *}, \tilde{X}_{b}^{n *}\right), b=1, \ldots, B$. Each $\quad 13$ sample consists of $n$ observations generated from empirical distribution $\hat{F}$ with replacement. $\quad 14$

Step 2. Construct $\Psi_{X_{b}^{n *}, k}$ and $\Psi_{\tilde{X}_{b}^{n *}, k}$ based on $\left(X_{b}^{n *}, \tilde{X}_{b}^{n *}\right), b=1, \ldots, B$.

Step 3. For each pair, $\Psi_{X_{b}^{n *}, k}$ and $\Psi_{\tilde{X}_{b}^{n *}, k}$, calculate their empirical clustering distance 16

$d_{\hat{F}}\left(\Psi_{X_{b}^{n *}, k}, \Psi_{\tilde{X}_{b}^{n *}, k}\right)=\frac{1}{n^{2}} \sum_{i=1}^{n} \sum_{j=1}^{n}\left|I\left\{\Psi_{X^{n *}, k}\left(x_{i}\right)=\Psi_{X^{n *}, k}\left(x_{j}\right)\right\}-I\left\{\Psi_{\tilde{X}^{n *}, k}\left(x_{i}\right)=\Psi_{\tilde{X}^{n *}, k}\left(x_{j}\right)\right\}\right|$.

Then the clustering instability $s(\Psi, k, n)$ can be estimated by

17

$$
\hat{s}_{B}(\Psi, k, n)=\frac{1}{B} \sum_{b=1}^{B} d_{\hat{F}}\left(\Psi_{X_{b}^{n *}, k}, \Psi_{\tilde{X}_{b}^{n *}, k}\right) .
$$


Step 4. Finally, the optimal number of clusters can be estimated by

$$
\hat{k}=\hat{k}(n)=\operatorname{argmin}_{2 \leq k \leq K} \hat{s}_{B}(\Psi, k, n) .
$$

Hereafter the maximum number of clusters $K$ is set as 10 . However, the selection of $K{ }_{2}$ depends on one's interest and should be adjusted according to the data. If $\hat{k}=K$, that is 3 the instability achieves its minimum at the boundary, we should increase the value of $K$. ${ }_{4}$

Algorithm 1 is demonstrated in Figure 1, the schematic diagram of the bootstrap method, $\quad 5$ where " $\rightarrow$ " means data generating and " $\Rightarrow$ " means estimating. This diagram is similar to 6 Figure 8.1 in Efron and Tibshirani (1993).

Insert Figure 1 about here

Furthermore, using the bootstrap, we can estimate the standard error of the estimated 9 clustering instability. The following algorithm describes a straightforward bootstrap proce- 10 dure, and some other more sophisticated procedures are discussed in Section 4.

Algorithm 2: Bootstrap method for estimating the standard error $\quad 12$

Step 1. Generate $C$ independent bootstrap samples $X_{c}^{n *}, c=1, \ldots, C$. Each sample 13 consists of $n$ observations generated from empirical distribution $\hat{F}$ with replacement. 14

Step 2. For sample $X_{c}^{n *}$, calculate the estimated clustering instability $\hat{s}_{B}^{c *}(\Psi, k, n)$ based 15 on $B$ bootstrap samples generating from $X_{c}^{n *}$. 16

Step 3. Calculate the sample standard deviation of $\hat{s}_{B}^{c *}(\Psi, k, n), c=1, \cdots, C$, which 17 estimates the standard error of the estimated clustering instability, $\hat{s}_{B}(\Psi, k, n) . \quad \square \quad 18$

Ideally, $\hat{s}_{\infty}(\Psi, k, n)=\theta(\hat{F})$. In practice, based on our limited experience, moderate large $\quad 19$ $B$, say $B=20$ or 50 , can result in very stable estimate of $s(\Psi, k, n)$ and then very stable $\quad 20$ estimate of $k_{0}$. Efron and Tibshirani (1993, Section 6.4) gave some rules of thumb for selecting ${ }_{21}$ the number of replications $B$, and demonstrated that the selection of $B$ can be guided by the ${ }_{22}$ 
coefficient of variation. Note that we can apply Algorithm 2 to estimate the standard error $\quad 1$ used in calculation of the coefficient of variation.

\subsection{The bootstrap estimates of the instability paths}

Although the clustering instability has attracted increasing attention, its theoretical justifi- $\quad 4$ cation remains unclear. One important issue, as pointed out in Krieger and Green (1999) and 5 Ben-David et al. (2006), is that any clustering algorithm is asymptotically stable as long as 6 it can be formulated as an optimization problem with a certain objective function that has a 7 unique global minimizer. On the other hand, it has been noted that although the instability 8 measures may converge to zero, the rates of convergence can behave differently when different 9 numbers of clusters are specified (e.g., Shamir and Tishby, 2007). 10

Denote the instability path for clustering algorithm $\Psi(\cdot ; k)$ as $\{s(\Psi, k, N): N \geq n\}$. To $\quad 11$ estimate $s(\Psi, k, N)$ for any given $N$ and then visualize the convergence path of the clustering 12 instability for any given number of clusters, we modify Algorithm 1 in what follows. The ${ }_{13}$ same idea has been used in Efron and Tibshirani (1993, Section 25.4) for power calculation. $\quad 14$

Algorithm 3: Bootstrap method for estimating the instability paths $\quad 15$

Step 1. Generate $B$ independent bootstrap sample-pairs $\left(X_{b}^{N *}, \tilde{X}_{b}^{N *}\right), b=1, \ldots, B$. Each $\quad 16$ sample consists of $N$ observations generated from empirical distribution $\hat{F}$ with replacement. $\quad{ }_{17}$

Step 2. Construct $\Psi_{X_{b}^{N *}, k}$ and $\Psi_{\tilde{X}_{b}^{N *}, k}$ based on $\left(X_{b}^{N *}, \tilde{X}_{b}^{N *}\right), b=1, \ldots, B . \quad 18$

Step 3. For each pair, $\Psi_{X_{b}^{N *}, k}$ and $\Psi_{\tilde{X}_{b}^{N *}, k}$, calculate empirical distance $d_{\hat{F}}\left(\Psi_{X_{b}^{N *}, k}, \Psi_{\tilde{X}_{b}^{N *}, k}\right)$. ${ }^{19}$ Then estimate $s(\Psi, k, N)$ by $\hat{s}_{B}(\Psi, k, N)=\frac{1}{B} \sum_{b=1}^{B} d_{\hat{F}}\left(\Psi_{X_{b}^{N *}, k}, \Psi_{\tilde{X}_{b}^{N *}, k}\right) . \quad \square \quad 20$

\section{Numerical results 21}

\subsection{An illustrative example 22}

We first examine an illustrative example in great detail. The simulated sample contains 23 three clusters in a two-dimensional space, each with size 50 and sampled from bivariate 24 
normal distributions with a common identity covariance matrix and distinct centers $(2,0), \quad 1$ $(-1,2)$, and $(-1,-2)$, respectively. Therefore, the sample size is $n=150$ and the optimal $\quad 2$ number of clusters is $k_{0}=3$. An observed sample is displayed in the left panel of Figure $2 . \quad 3$ Insert Figure 2 about here

For this observed sample, we apply both the newly proposed bootstrap method and the 5 cross-validation method to select the number of clusters. (Here the cross-validation method 6 is the one based on averaging, that is $\mathrm{CV}_{a}$ in Wang (2010), where the cross-validation method 7 based on voting, $\mathrm{CV}_{v}$, was also proposed.) In the bootstrap method, 50 bootstrap sample- 8 pairs are generated, while in the cross-validation method, 50 data splittings are performed 9 and the splitting ratio is set as $1 / 3$ (one third as the validation dataset, the other two thirds 10 as two training datasets). Here the k-means algorithm is applied. Both methods produce 11 estimates of the clustering instability for $k=2, \ldots, 10$. The results are displayed in the 12 right panel of Figure 2. Both methods select the optimal number of clusters as $\hat{k}=3$, which 13 correctly estimates the true optimal number of clusters. 14

From the right panel of Figure 2, we also find that the cross-validation estimated in- ${ }_{15}$ stabilities are consistently larger than those estimated using the bootstrap method, because 16 the sizes of two training datasets in the cross-validation method are only $n / 3$. Actually, the 17 cross-validation only provides estimates for $s(\Psi, k, n / 3)$ instead of $s(\Psi, k, n)$, whereas the 18 bootstrap method provides nonparametric MLE for $s(\Psi, k, n)$. 19

Figure 2 also displays the estimated standard errors, shown as the bars. Here the standard 20 errors are estimated using Algorithm 2 with $C=100$. It is found that the standard errors ${ }_{21}$ in the bootstrap method are smaller than those in the cross-validation method, due to the 22 same reason that the effect simple size in the bootstrap method is larger. 23

We go further to see if the bootstrap distribution of $d_{\hat{F}}\left(\Psi_{X^{n *}, k}, \Psi_{\tilde{X}^{n *}, k}\right)$ is similar to the 24 true distribution of $d_{F}\left(\Psi_{X^{n}, k}, \Psi_{\tilde{X}^{n}, k}\right)$. We only consider the case where $k$ is preset as 2.25 
For this aim, we generate 1000 bootstrap sample-pairs $\left(X_{b}^{n *}, \tilde{X}_{b}^{n *}\right)$ from the given empirical 1 distribution $\hat{F}, b=1, \ldots, 1000$. Then the clustering distances $d_{\hat{F}}\left(\Psi_{X_{b}^{n *, 2}}, \Psi_{\tilde{X}_{b}^{n *, 2}}\right)$ are calcu- 2 lated. On the other hand, we generate 1000 random sample-pairs $\left(X_{b}^{n}, \tilde{X}_{b}^{n}\right)$ from the true $\quad 3$ simulation distribution $F, b=1, \ldots, 1000$. Then the clustering distances $d_{F}\left(\Psi_{X_{b}^{n}, 2}, \Psi_{\tilde{X}_{b}^{n}, 2}\right)$ are calculated using Mente Carlo method based on 50 newly generated copies of $X^{n}$. The ${ }_{5}$ histograms of $\left\{d_{\hat{F}}\left(\Psi_{X_{b}^{n *}, 2}, \Psi_{\tilde{X}_{b}^{n *}, 2}\right), b=1, \ldots 1000\right\}$ and $\left\{d_{F}\left(\Psi_{X_{b}^{n}, 2}, \Psi_{\tilde{X}_{b}^{n}, 2}\right), b=1, \ldots, 1000\right\}$ are displayed respectively in Figure 3.

$\overline{\overline{\text { Insert Figure } 3 \text { about here }}}$

From Figure 3, we find that the two histograms are similar to each other; both of them 9 are in U-shape, with large frequencies at the two ends and small frequencies in the between. $\quad 10$ Also, the means and the standard deviations of these two histograms are very close. Noting 11 that the two means are respectively the bootstrap estimate and the Monte Carlo estimate of 12 the true clustering instability $s(\Psi, 2, n)$, we are further assured that the bootstrap method 13 gives almost unbiased estimates of the clustering instabilities. 14

\subsection{Four simulation examples in Wang (2010) 15}

We examine the newly proposed bootstrap method using the same four simulation examples 16 as in Wang (2010). The first example consists of two elongated clusters in a three-dimensional 17 space, each of size 100. This is also the example (e) in Tibshirani et al. (2001). Each cluster 18 is generated as follows. Set $x_{1}=x_{2}=x_{3}=t$ with $t$ taking 100 equally spaced values from $\quad 19$ -0.5 to 0.5 and then Gaussian noise with standard deviation 0.1 is added to each feature. 20 Cluster 2 is generated in the same way, except that the value 10 is added to each feature at ${ }^{21}$ the end. The second example contains four non-Gaussian clusters in a ten-dimensional space, 22 each of size 100. The clusters reside in a two-dimensional subspace, and are sampled from 23 bivariate exponential distributions with location parameters $(4,4),(4,-4),(-4,4),(-4,-4) \quad 24$ 
and a common scale parameter 1 , and the remaining eight dimensions are noises sampled 1 from standard exponential distribution. The other two are non-distance based examples, the 2 two-moon example and the bull's eye example, whose two realizations are displayed in Figure 3 4 (a) and (b) respectively. Wang (2010) compared his two cross-validation methods with six 4 other methods and concluded that the cross-validation based on averaging, $\mathrm{CV}_{a}$, performs 5 the best in these four simulation examples. 6

We perform the k-means algorithm for the first two examples (distance-based) and the 7 spectral clustering algorithm (Ng et al., 2001) for the other two examples (non-distance- 8 based). The k-means algorithm assigns each observation to the cluster whose center is nearest, 9 and the center is the average of all the observations in the cluster. The spectral clustering 10 algorithm defines a similarity matrix first and then makes use of the spectrum of the similarity 11 matrix to perform principle component analysis for clustering in fewer dimensions. 12

We compare the bootstrap method with $\mathrm{CV}_{a}$, along with three recent methods, the ${ }_{13}$ silhouette statistic in Kaufman and Rousseeuw (1990), the gap statistic in Tibshirani et ${ }_{14}$ al. (2001), and the jump statistic in Sugar and James (2003). Kaufman and Rousseeuw 15 (1990) defined the silhouette statistic as $s(k)=n^{-1} \sum_{i=1}^{n}\left(b\left(x_{i}\right)-a\left(x_{i}\right)\right) / \max \left\{a\left(x_{i}\right), b\left(x_{i}\right)\right\}, \quad 16$ where $a\left(x_{i}\right)$ is the averaged distance to other observations in its cluster, and $b\left(x_{i}\right)$ is the 17 averaged distance to observations in its nearest cluster. Tibshirani et al. (2001) defined the 18 gap statistic as $\operatorname{Gap}(k)=B^{-1} \sum_{b=1}^{B} \log \left(W_{k}^{* b}\right)-\log \left(W_{k}\right)$, where $B$ reference datasets are 19 generated and $W_{k}$ and $W_{k}^{* b}$ are the within-cluster sums of squares from the observed dataset 20 and the $b$ th reference dataset respectively. Sugar and James (2003) defined the jump statistic ${ }_{21}$ as $\operatorname{Jump}(k)=\hat{d}_{k}^{-p / 2}-\hat{d}_{k-1}^{-p / 2}$, where $\hat{d}_{k}=p^{-1} \sum_{i=1}^{n} \min _{c_{r}}\left(x_{i}-c_{r}\right)^{\prime}\left(x_{i}-c_{r}\right)$. The number of 22 clusters is chosen such that $s(k), \operatorname{Gap}(k)$ or $\operatorname{Jump}(k)$ is maximized. 23

Each simulation example is repeated 50 times and the results are summarized in Table $1 . \quad 24$ Here the number of bootstrap sample-pairs in the bootstrap method, the number of random ${ }_{25}$ 
splits in the cross-validation method, and the number of reference datasets are all set as $50 . \quad 1$

Insert Table 1 about here

From Table 1, we find that the bootstrap method performs as well as the cross-validation 3 method for selecting the number of clusters, and clearly outperforms the others. The gap 4 statistic also performs very well in Example 1, which is of Gaussian distribution, and in the 5 two-moon example. However, the gap statistic does not perform well in Example 2, where 6 the clusters are non-spherical, and is not working in the bull's eye example, one of the non- 7 distance based examples. Neither the silhouette statistic nor the jump statistic performs 8 well here. Because the sample sizes in these examples are in hundreds, the advantage of the 9 bootstrap method over the cross-validation method is not clear. However, as byproducts, the 10 bootstrap method can provide almost unbiased estimates for the clustering stabilities and 11 the stability paths. The latter will be further demonstrated by two real examples. 12

\subsection{More simulation examples}

To further compare the bootstrap method with the cross-validation method and the gap 14 statistic, we revisit the illustrative example but under various conditions, such as the true 15 optimal number of clusters, dimension of features, correlation among features, and clusters 16 overlapping level.

Two groups of simulation settings are considered. In the first group, there are 3 clusters, 18 that is $k_{0}=3$, while in the second group, $k_{0}=5$. Each cluster contains 50 observations, and 19 the number of features $p$ is either 2 or 5 . We describe the first two dimensions as follows. In 20 the first group, the three cluster centers are on the vertices of an equilateral triangle with the ${ }_{21}$ edge length $d$. In the second group, four cluster centers are on the vertices of a square and 22 the fifth cluster center is at the middle with the diagonal length $2 d$. For the settings where ${ }_{23}$ $p=5$, the remaining three dimensions contain no clustering information. 24 
The observations are generated from multivariate normal distributions with means being $\quad 1$ their corresponding centers, variances 1 , and correlation coefficients $\rho$, which is equal to 2 either 0 or 0.6 . The length $d$, which controls the clusters overlapping level, is set as 3 , 4 , or 6 . 3 When $d=6$, clusters are moderately overlapped, while $d=4$ indicates serious overlapping $\quad 4$ and $d=3$ indicates extremely serious overlapping. $\quad 5$

Each simulation setting is repeated 50 times and the results are presented in Table $2 . \quad 6$ Again, the k-means is performed, the number of bootstrap sample-pairs in the bootstrap 7 method, the number of random splits in the cross-validation method, and the number of 8 reference datasets are all set as 50. The maximum number of clusters $K$ is set as $10 . \quad 9$

Insert Table 2 about here

From Table 2, we find that the bootstrap method performs slightly better than the cross- $\quad 11$ validation method. In the settings where $p=2$, all the three methods performs very well 12 even when the clusters are seriously overlapped, and the correlation between variables does 13 not cause any problem. 14

Both the stability selection methods are outperformed by the gap statistic. This is not 15 surprising because here the data are generated from Gaussian distribution and the gap statis- $\quad 16$ tic is based on the Euclidean distance. In the previous subsection, it has shown that the gap 17 statistics works well with Gaussian clusters or other spherical distributed clusters, but it fails 18 when the clusters are non-spherical. $\quad 19$

In the settings where $p=5$ and three features contain no clustering information, no 20 method is working except in the settings where $k_{0}=3$ and $\rho=0$. This indicates that it is ${ }^{21}$ necessary to consider some feature selection procedure to get rid of redundant features before 22 conducting cluster analysis; for example, Witten and Tibshirani (2010) proposed a general ${ }_{23}$ sparse clustering framework and Sun, Wang, and Fang (2011) proposed a regularized k-means $\quad 1$ algorithm for high-dimensional data. 2 
In the settings where $p=5, k_{0}=3$, and $\rho=0$, the gap statistic is working with both 3 $d=4$ and $d=6$, while the bootstrap method is working only when $d=6$ and the cross- $\quad 4$ validation method is not working. This indicates that the stability selection methods are not 5 working well for the cases where clusters are seriously overlapped.

\subsection{Two real data examples}

We examine the effectiveness of the bootstrap method in two real data examples, comparing 8 it with the cross-validation method in Wang (2010). The first one is iris data (Fisher, 1936), 9 which contains 150 observations from three different species of iris, each with four measures 10 on the length and width of the sepal and petal. The second one is wine data (Aeberhard, 11 Coomans and de Vel, 1992), which consists of 12 measurements for each of 178 alcohols 12 from three different kinds. The iris dataset is available in R, and the wine dataset is down- ${ }_{13}$ loaded from University of California Irvine machine learning repository. Figure 5 displays 14 the scatterplots of these two datasets.

In both real examples, the true numbers of clusters are unknown, but their available class 16 memberships provide a helpful reference from the true clustering. The results are displayed 17 in Figure 6 and Figure 7 respectively. In both examples, k-means is performed, 50 bootstrap 18 sample-pairs are generated for the bootstrap method, and 50 data-splittings with ratio $1 / 3 \quad 19$ are generated for the cross-validation method. 20

In the iris example, from the left panel of Figure 6, both the bootstrap and cross-validation $\quad 21$ methods select the optimal number of clusters as $\hat{k}=2$, while the dataset contains three ${ }_{22}$ species of iris. Similar observation was made in Sugar and James (2003). This example shows ${ }_{23}$ the weakness of the stability selection methods when some clusters are seriously overlapped, 24 which has already been shown in Subsection 3.3. We also see that the bootstrap estimated 1 instabilities are larger than those by the cross-validation method for the same reason given 2 in Section 4. From the right panel of Figure 6, we see that the instability path of $k=2 \quad 3$ 
converges to zero the fastest.

Insert Figure 6 about here

In the wine example, from the left panel of Figure 7 , both the bootstrap and cross- 6 validation methods select the optimal number of clusters as $\hat{k}=3$, which agrees with the 7 number of classes in the dataset. Similarly, the bootstrap estimated instabilities are larger 8 than those by the cross-validation method. And from the right panel of Figure 7, we see that 9 the instability path of $k=3$ converges to zero much faster than the other two. 10

Insert Figure 7 about here

\section{Discussion}

We propose the bootstrap method for estimating the clustering instability and then selecting $\quad 13$ the number of clusters. It can be applied to any distance-based or non-distance-based clus- 14 tering algorithm. It is an alternative to the cross-validation method in Wang (2010), whose 15 selection consistency has been established. Since the bootstrap method provides nonpara- 16 metric MLE of the clustering instability, which are believed to enjoy many good properties, 17 we don't attempt to work on the selection consistency of the bootstrap method. 18

The main goal in this manuscript is selecting the number of clusters, but the method 19 can also be used to construct confidence interval estimate for the clustering instability. The 20 jackknife-after-bootstrap method (Efron, 1992), the $\mathrm{BC}_{a}$ (Efron, 1987) and the $\mathrm{ABC}$ (DiCi- ${ }_{21}$ ccio and Efron, 1992) methods can provide desirable confidence interval estimates. 22

The bootstrap method has other applications rather than selection of the number of ${ }_{23}$ clusters. For example, the bootstrap method can also be applied to select tuning parameters $\quad 1$ in the regularized k-means (Sun et al., 2011), the penalized cluster analysis (Fang and Wang, 2 2011) and the sparse clustering (Witten and Tibshirani, 2010). 3 


\section{Acknowledgement}

We thank the Editor, an Associate Editor and two referees for their constructive comments 5 that improve the manuscript significantly.

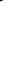

\section{References}

[1] Aeberhard, S., Coomans, D., and de Vel, O. (1992). Comparison of classifiers in high 8 dimensional settings, Technical Report, 92-02, Department of Computer Science and 9 Department of Mathematics and Statistics, James Cook University of North Queensland. $\quad 10$

[2] Ben-David, S., von Luxburg, U., and Pal, D. (2006). A sober look at stability of cluster- ${ }_{11}$ ing, 19th Annual Conference on Learning Theory (COLT 2006).

[3] Ben-Hur, A., Elisseeff, A., and Guyon, I. (2002). A stability based method for discovering 13 structure in clustered data, Pacific Symposium on Biocomputing 7: 6-17.

[4] Bryant, P. (2002). More on the stability of hierarchical clustering, Paper presented at the ${ }_{15}$ Classification Society of North America Meeting, Madison, WI.

[5] Calinski, R. B. and Harabasz, J. (1974). A dendrite method for cluster analysis, Com- ${ }_{17}$ munications in Statistics - Simulation and Computation 3: 1-27.

[6] Cheng, R. and Milligan, G. W. (1996). K-means clustering methods with influence de- ${ }_{19}$ tection, Educational and Psychological Measurement 56: 833-838.

[7] Efron, B. (1987). Better bootstrap confidence intervals (with discussion), Journal of ${ }^{21}$ American Statistical Association 82: 171-200.

[8] Efron, B. (1992). Jackknife-after-bootstrap standard errors and influence functions, Jour- $\quad 1$ nal of Royal Statistical Society, Series B 54: 83-127.
12

3

15


[9] DiCiccio, T. J. and Efron, B. (1992). More accurate confidence intervals in exponential 3 families, Biometrika 79: 231-245.

[10] Efron B. and Tibshirani, R. J. (1993). An Introduction to the Bootstrap, Chapman \& 5 Hall/CRC.

[11] Fang, Y. and Wang, J. (2011). Penalized cluster analysis with applications to family 7 data, Computational Statistics and Data Analysis 55: 2128-2136.

[12] Fisher, R. A. (1936). The use of multiple measurements in taxonomic problems, Annals 9 of Eugenics 7: 179-188.

[13] Fowlkes, E. B. and Mallows, C. L. (1983). A method for comparing two hierachical 11 clusterings. Journal of the American Statistical Association 78: 553-584.

[14] Gnanadesikan, R. (1997). Methods for Statistical Data Analysis of Multivariate Obser- ${ }_{13}$ vations, 2nd Edition, John Wiley \& Sons, Inc., New York.

[15] Hartigan, J. A. (1975). Clustering Algorithms, Wiley, New York.

[16] Johnson, S. C. (1967). Hierarchical Clustering Schemes, Psychometrika 2: 241-254. 16

[17] Kaufman, L. and Rousseeuw, P. (1990). Finding Groups in Data: An introduction to ${ }_{17}$ Cluster Analysis, Wiley, New York.

[18] Krieger, A. W. and Green, P. E. (1999). A cautionary note on using internal crossvali- 19 dation to select the number of clusters. Psychometrika 64: 341-353.

[19] Krzanowski, W. J. and Lai, Y. T. (1985). A criterion for determining the number of ${ }_{21}$ clusters in a data set, Biometrics 44: 23-34.

[20] Lange, T., Roth, V., Braun, M., and Buhmann, J. (2004). Stability-based validation of 2 clustering solutions, Neural Computation 16: 1299-1323. 
[21] MacQueen, J. B. (1967). Some methods for classification and analysis of multivariate 4 observations, Proceedings of 5-th Berkeley Symposium on Mathematical Statistics and 5 Probability, Berkeley, University of California Press, 1: 281-297.

[22] McIntyre, R. M. and Blashfield, R. K. (1980). A nearest-centroid technique for evaluating 7 the minimum variance clustering procedure, Multivariate Behavioral Research 15: 225- 8 238.

[23] Milligan, G. W. and Cooper, M. C. (1985). An examination of procedures for determining 10 the number of clusters in a dataset, Psychometrika 50: 159-179.

[24] Ng, A., Jordan, M. and Weiss, Y. (2001). On spectral clustering: analysis and an algo- ${ }_{12}$ rithm. In Adv. Neural. Info. Processing Sys. (NIPS2001), Ed. T. Dietterich, S. Becker 13 and Z. Ghahramani, pp. 84956. Cambridge: MIT Press 14

[25] Shi, J. and Malik, J. (2000). Normalized cuts and image segmentation, IEEE Transac- ${ }_{15}$ tions on Pattern Analysis and Machine Intelligence 22: 888-905. 16

[26] Shamir, O. and Tishby, T. (2007). Cluster stability for finite samples. In Adv. Neural ${ }_{17}$ Info. Processing Sys. (NIPS2007), Ed. J. Platt, D. Koller, Y. Singer and S. Roweis, pp. 18 1297-1304. Cambridge: MIT Press. 19

[27] Steinley, D. (2008). Stability analysis in K-means clustering, British Journal of Mathe- 20 matical and Statistical Psychology 61: 255-273. 21

[28] Sugar, C. and James, G. (2003). Finding the number of clusters in a data set: an 22 imformation theoretic approach, Journal of American Statistical Association 98: 750- 23 763.

[29] Sun, W., Wang, J. and Fang, Y. (2011). Regularized k-means clustering of high- ${ }_{2}$ dimensional data and its asymptotic consistency. Manuscript. 
[30] Tibshirani, R., Walther, G., and Hastie, T. (2001). Estimating the number of clusters 4 in a data set via the gap statistic, Journal of Royal Statistical Society, Series B, 63: 373 511-528.

[31] Wang, J. (2010). Consistent selection of the number of clusters via cross-validation, 375 Biometrika 97: 893-904.

[32] Witten, D. M. and Tibshirani, R. (2010). A framework for feature selection in clustering, 377 Journal of the American Statistical Association 105: 713-726. 
Table 1: Four simulation examples in Wang (2010)

\begin{tabular}{|c|c|c|c|c|c|c|c|c|c|}
\hline \multirow{2}{*}{ Method } & \multicolumn{9}{|c|}{ Estimated number of clusters } \\
\hline & 2 & 3 & 4 & 5 & 6 & 7 & 8 & 9 & 10 \\
\hline \multicolumn{10}{|c|}{ Example 1: Two elongated clusters in 3 dimensions } \\
\hline Silhouette & 50 & 0 & 0 & 0 & 0 & 0 & 0 & 0 & 0 \\
\hline Gap & 50 & 0 & 0 & 0 & 0 & 0 & 0 & 0 & 0 \\
\hline Jump & 0 & 0 & 0 & 0 & 4 & 0 & 36 & 7 & 3 \\
\hline $\mathrm{CV}_{a}$ & 50 & 0 & 0 & 0 & 0 & 0 & 0 & 0 & 0 \\
\hline Bootsrap & 50 & 0 & 0 & 0 & 0 & 0 & 0 & 0 & 0 \\
\hline \multicolumn{10}{|c|}{ Example 2: Four Exponential clusters in 10 dimensions } \\
\hline Silhouette & 0 & 1 & 36 & 8 & 4 & 1 & 0 & 0 & 0 \\
\hline Gap & 46 & 0 & 1 & 3 & 0 & 0 & 0 & 0 & 0 \\
\hline Jump & 0 & 0 & 50 & 0 & 0 & 0 & 0 & 0 & 0 \\
\hline $\mathrm{CV}_{a}$ & 0 & 0 & 50 & 0 & 0 & 0 & 0 & 0 & 0 \\
\hline Bootstrap & 0 & 0 & 50 & 0 & 0 & 0 & 0 & 0 & 0 \\
\hline \multicolumn{10}{|c|}{ The two-moon example } \\
\hline Silhouette & 0 & 0 & 1 & 2 & 9 & 5 & 13 & 13 & 7 \\
\hline Gap & 50 & 0 & 0 & 0 & 0 & 0 & 0 & 0 & 0 \\
\hline Jump & 39 & 0 & 0 & 3 & 0 & 3 & 1 & 3 & 1 \\
\hline $\mathrm{CV}_{a}$ & 50 & 0 & 0 & 0 & 0 & 0 & 0 & 0 & 0 \\
\hline Bootstrap & 50 & 0 & 0 & 0 & 0 & 0 & 0 & 0 & 0 \\
\hline \multicolumn{10}{|c|}{ The bull's eye example } \\
\hline Silhouette & 0 & 0 & 6 & 9 & 2 & 5 & 10 & 9 & 9 \\
\hline Gap & 10 & 27 & 10 & 2 & 1 & 0 & 0 & 0 & 0 \\
\hline Jump & 0 & 4 & 12 & 11 & 4 & 9 & 3 & 4 & 3 \\
\hline $\mathrm{CV}_{a}$ & 50 & 0 & 0 & 0 & 0 & 0 & 0 & 0 & 0 \\
\hline Bootstrap & 50 & 0 & 0 & 0 & 0 & 0 & 0 & 0 & 0 \\
\hline
\end{tabular}


Table 2: More simulation examples

\begin{tabular}{|c|c|c|c|c|c|c|c|c|c|c|c|c|}
\hline \multirow{2}{*}{\multicolumn{4}{|c|}{ clusters }} & \multicolumn{4}{|c|}{$k_{0}=3$} & \multicolumn{5}{|c|}{$k_{0}=5$} \\
\hline & & & & 2 & 3 & 4 & $\geq 5$ & $\leq 3$ & 4 & 5 & 6 & $\geq 7$ \\
\hline \multirow{12}{*}{$p=2$} & \multirow{6}{*}{$d=3$} & \multirow{3}{*}{$\rho=0$} & Gap & 0 & 50 & 0 & 0 & 0 & 0 & 50 & 0 & 0 \\
\hline & & & $\mathrm{CV}_{a}$ & 0 & 41 & 4 & 5 & 0 & 0 & 39 & 2 & 1 \\
\hline & & & Boot & 0 & 48 & 0 & 2 & 0 & 9 & 38 & 2 & 1 \\
\hline & & \multirow{3}{*}{$\rho=0.6$} & Gap & 0 & 47 & 3 & 0 & 0 & 0 & 46 & 2 & 2 \\
\hline & & & $\mathrm{CV}_{a}$ & 0 & 40 & 5 & 5 & 0 & 3 & 40 & 3 & 4 \\
\hline & & & Boot & 0 & 41 & 4 & 5 & 0 & 4 & 41 & 3 & 2 \\
\hline & \multirow{6}{*}{$d=4$} & \multirow{3}{*}{$\rho=0$} & Gap & 0 & 50 & 0 & 0 & 0 & 0 & 50 & 0 & 0 \\
\hline & & & $\mathrm{CV}_{a}$ & 0 & 50 & 0 & 0 & 0 & 0 & 50 & 0 & 0 \\
\hline & & & Boot & 0 & 50 & 0 & 0 & 0 & 0 & 50 & 0 & 0 \\
\hline & & \multirow{3}{*}{$\rho=0.6$} & Gap & 0 & 50 & 0 & 0 & 0 & 0 & 50 & 0 & 0 \\
\hline & & & $\mathrm{CV}_{a}$ & 0 & 50 & 0 & 0 & 0 & 2 & 48 & 0 & 0 \\
\hline & & & Boot & 0 & 50 & 0 & 0 & 0 & 6 & 44 & 0 & 0 \\
\hline \multirow{12}{*}{$p=5$} & \multirow{6}{*}{$d=4$} & \multirow{3}{*}{$\rho=0$} & Gap & 0 & 35 & 9 & 6 & 4 & 1 & 9 & 19 & 17 \\
\hline & & & $\mathrm{CV}_{a}$ & 0 & 0 & 1 & 49 & 0 & 0 & 0 & 0 & 50 \\
\hline & & & Boot & 0 & 5 & 3 & 42 & 0 & 0 & 0 & 0 & 50 \\
\hline & & \multirow{3}{*}{$\rho=0.6$} & Gap & 0 & 1 & 3 & 46 & 0 & 4 & 2 & 17 & 30 \\
\hline & & & $\mathrm{CV}_{a}$ & 1 & 0 & 0 & 49 & 0 & 0 & 0 & 0 & 50 \\
\hline & & & Boot & 5 & 0 & 0 & 45 & 7 & 0 & 0 & 1 & 42 \\
\hline & \multirow{6}{*}{$d=6$} & \multirow{3}{*}{$\rho=0$} & Gap & 0 & 50 & 0 & 0 & 1 & 0 & 13 & 19 & 17 \\
\hline & & & $\mathrm{CV}_{a}$ & 0 & 26 & 17 & 7 & 0 & 0 & 0 & 0 & 50 \\
\hline & & & Boot & 0 & 50 & 0 & 0 & 0 & 0 & 0 & 0 & 50 \\
\hline & & \multirow{3}{*}{$\rho=0.6$} & Gap & 0 & 0 & 5 & 45 & 0 & 2 & 2 & 25 & 21 \\
\hline & & & $\mathrm{CV}_{a}$ & 0 & 0 & 1 & 49 & 0 & 0 & 0 & 0 & 50 \\
\hline & & & Boot & 1 & 0 & 5 & 44 & 3 & 0 & 0 & 2 & 45 \\
\hline
\end{tabular}


Figure 1: Schematic diagram of the bootstrap method

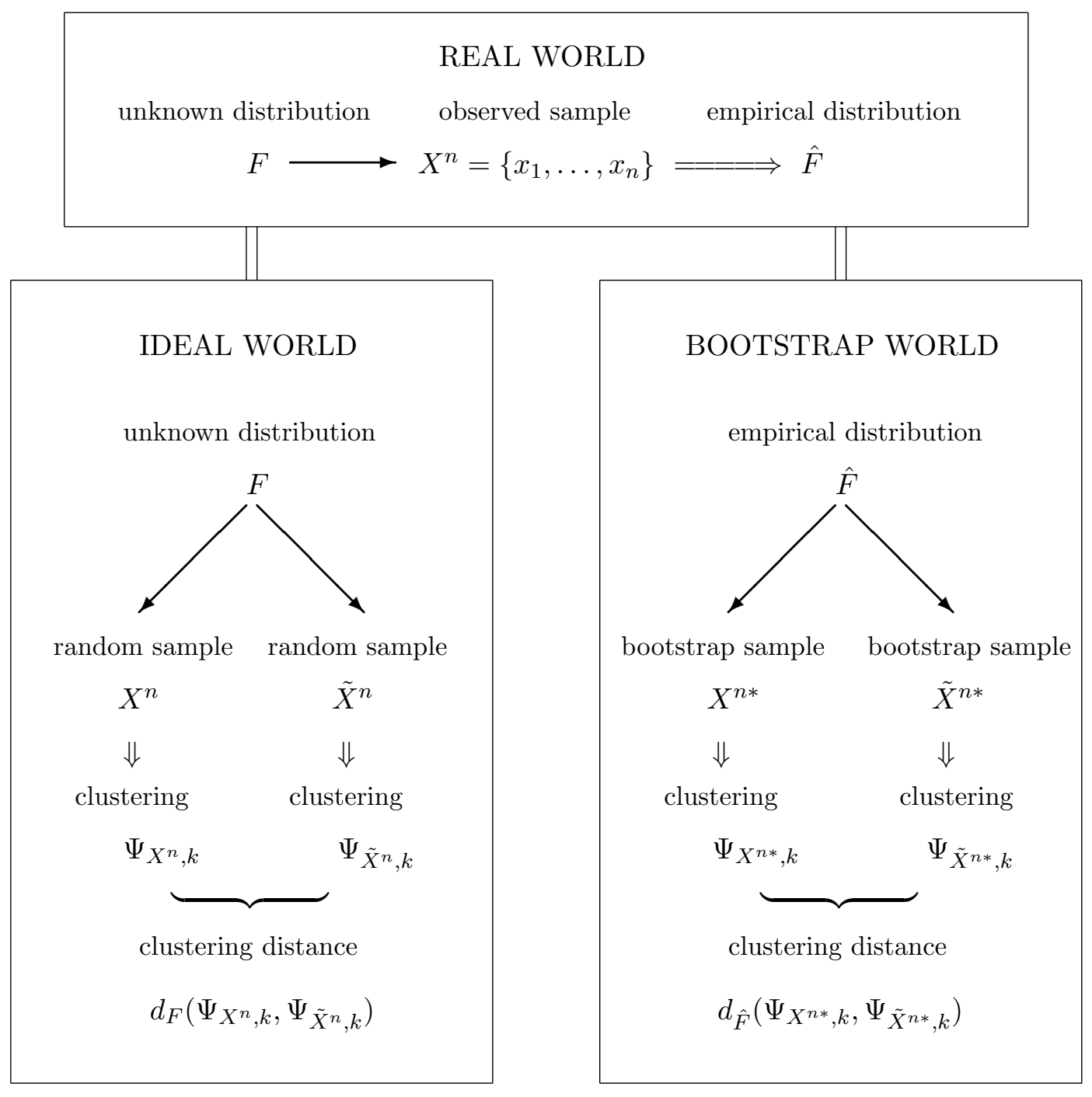


Figure 2: Illustrative example
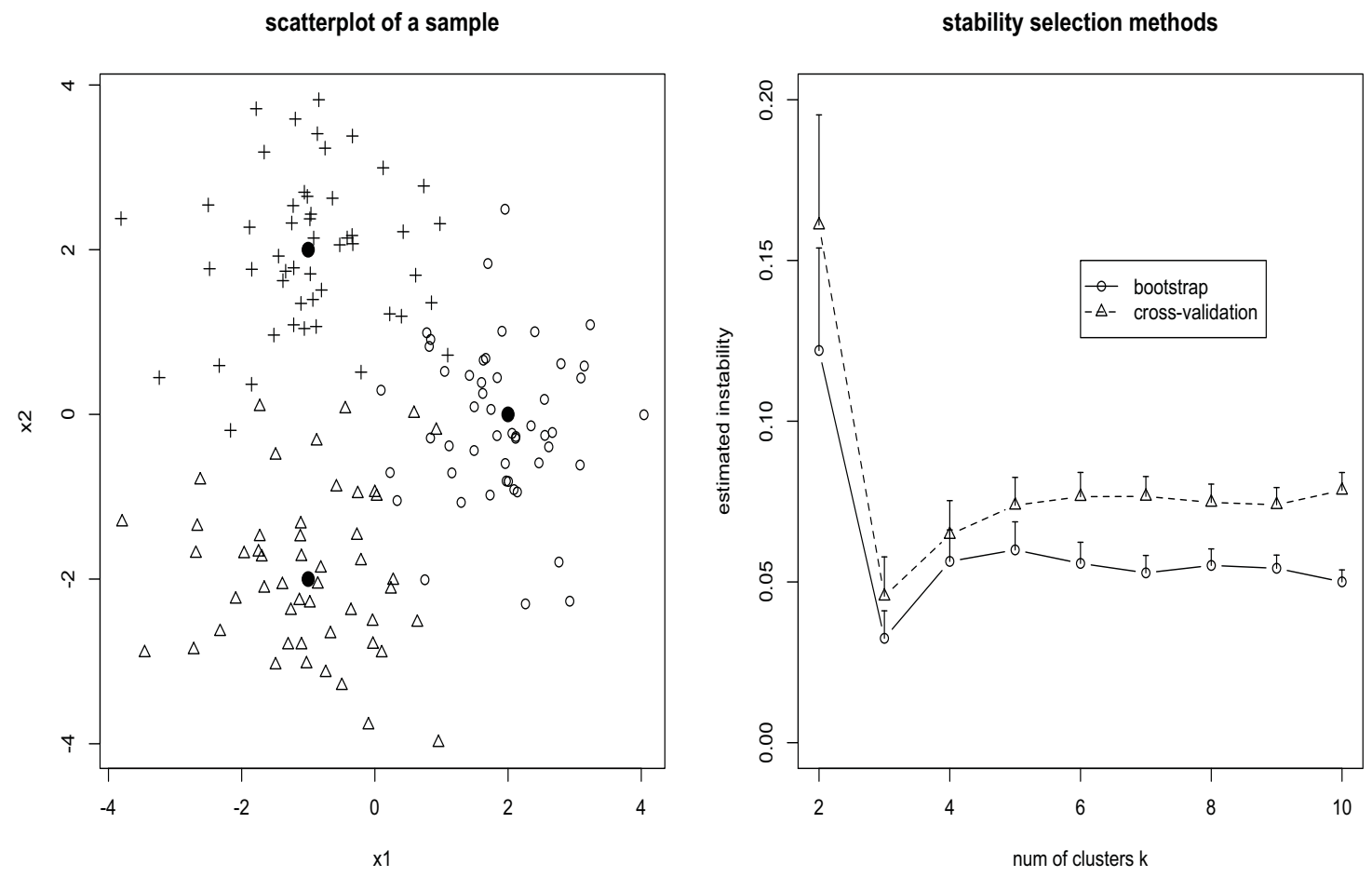
Figure 3: True distribution and bootstrap distribution of clustering distance
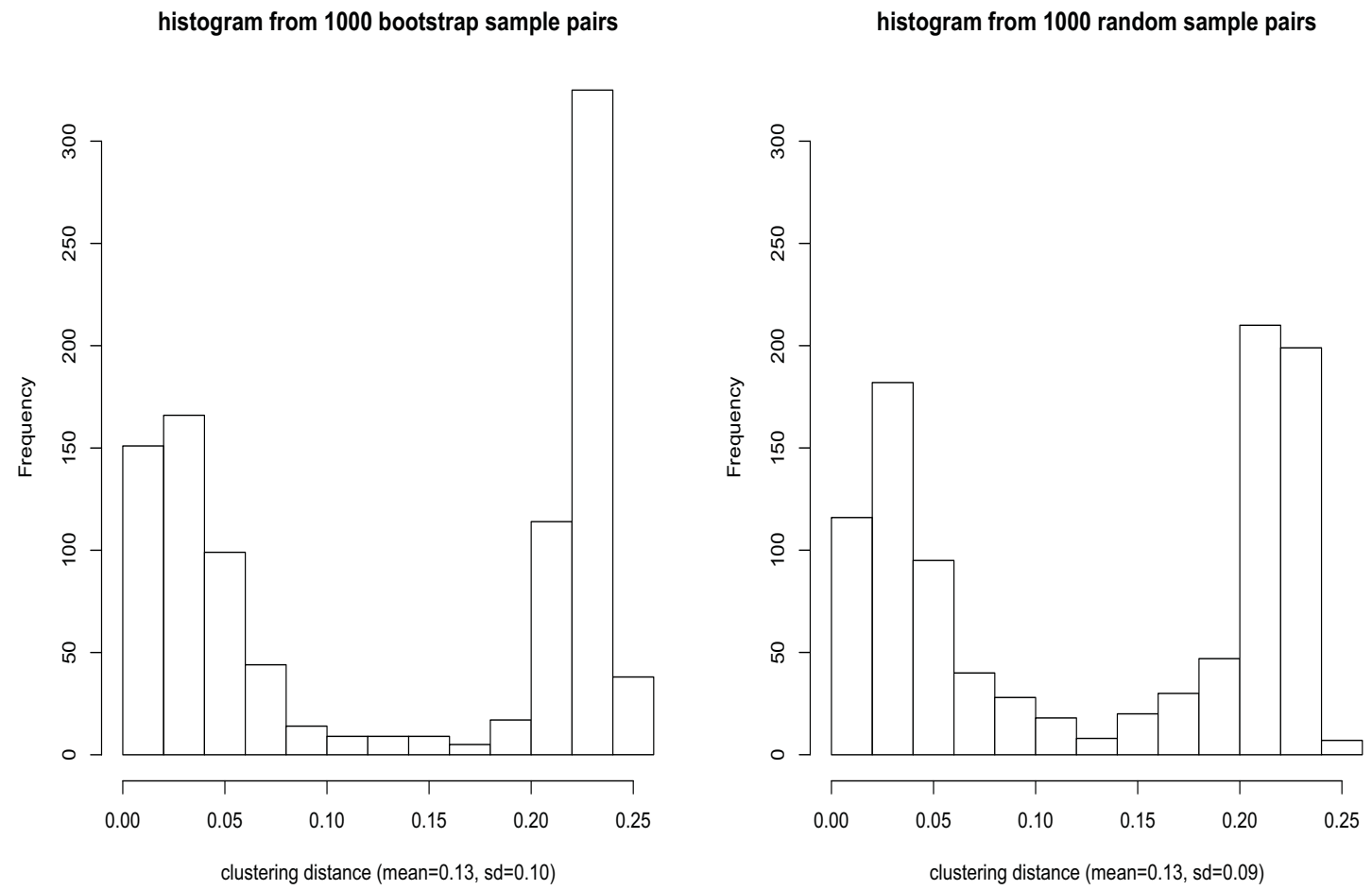
Figure 4: Some scatterplots

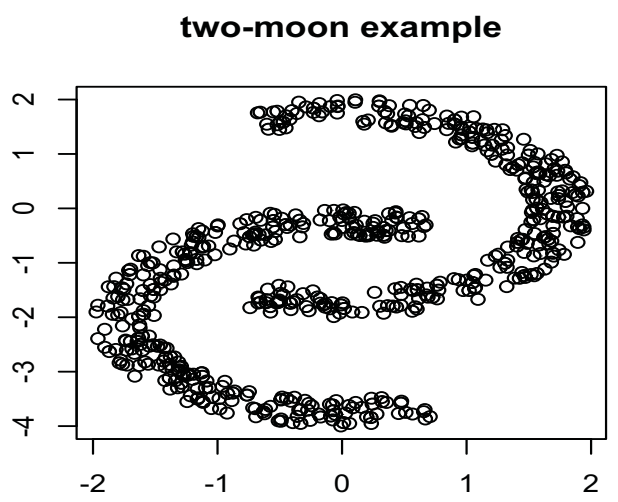

(a)

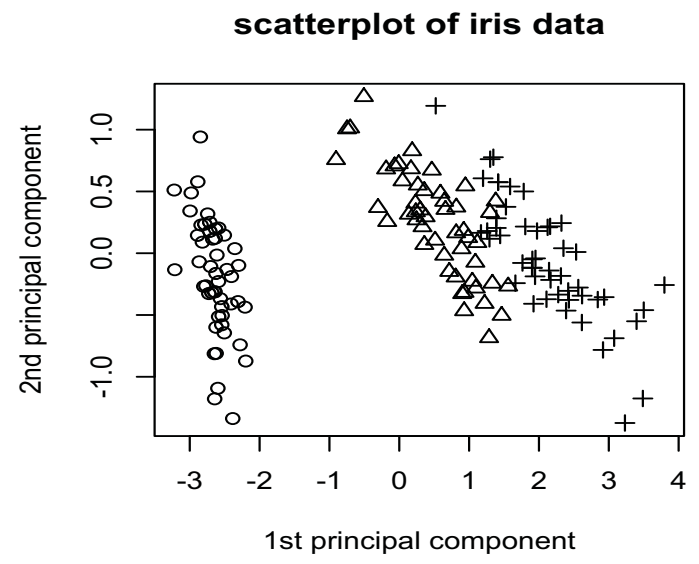

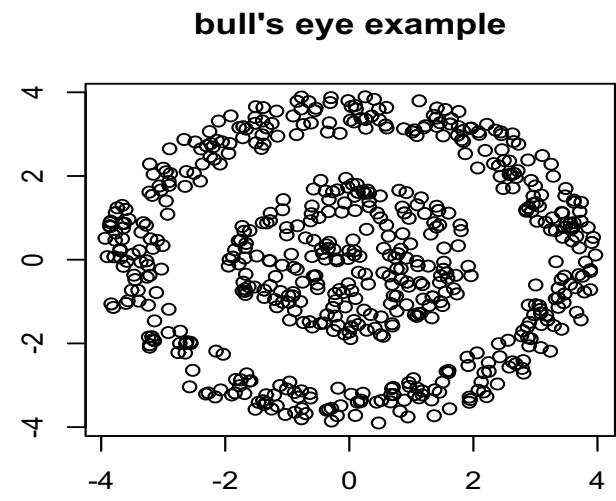

(b)

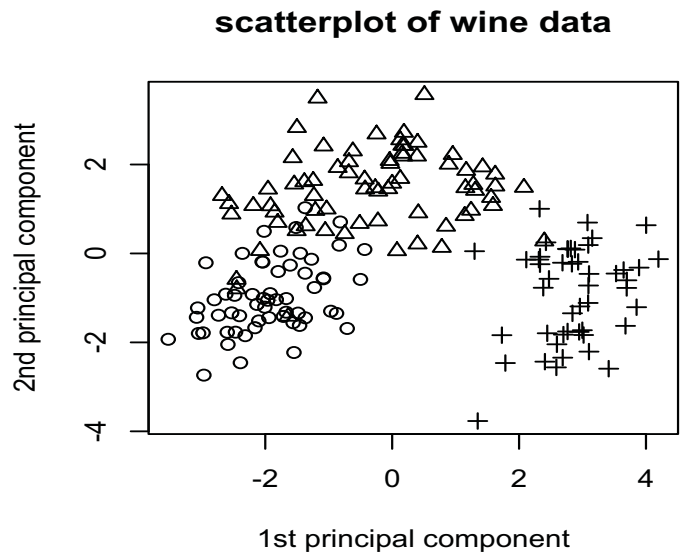


Figure 5: Iris data example $(\hat{k}=2)$
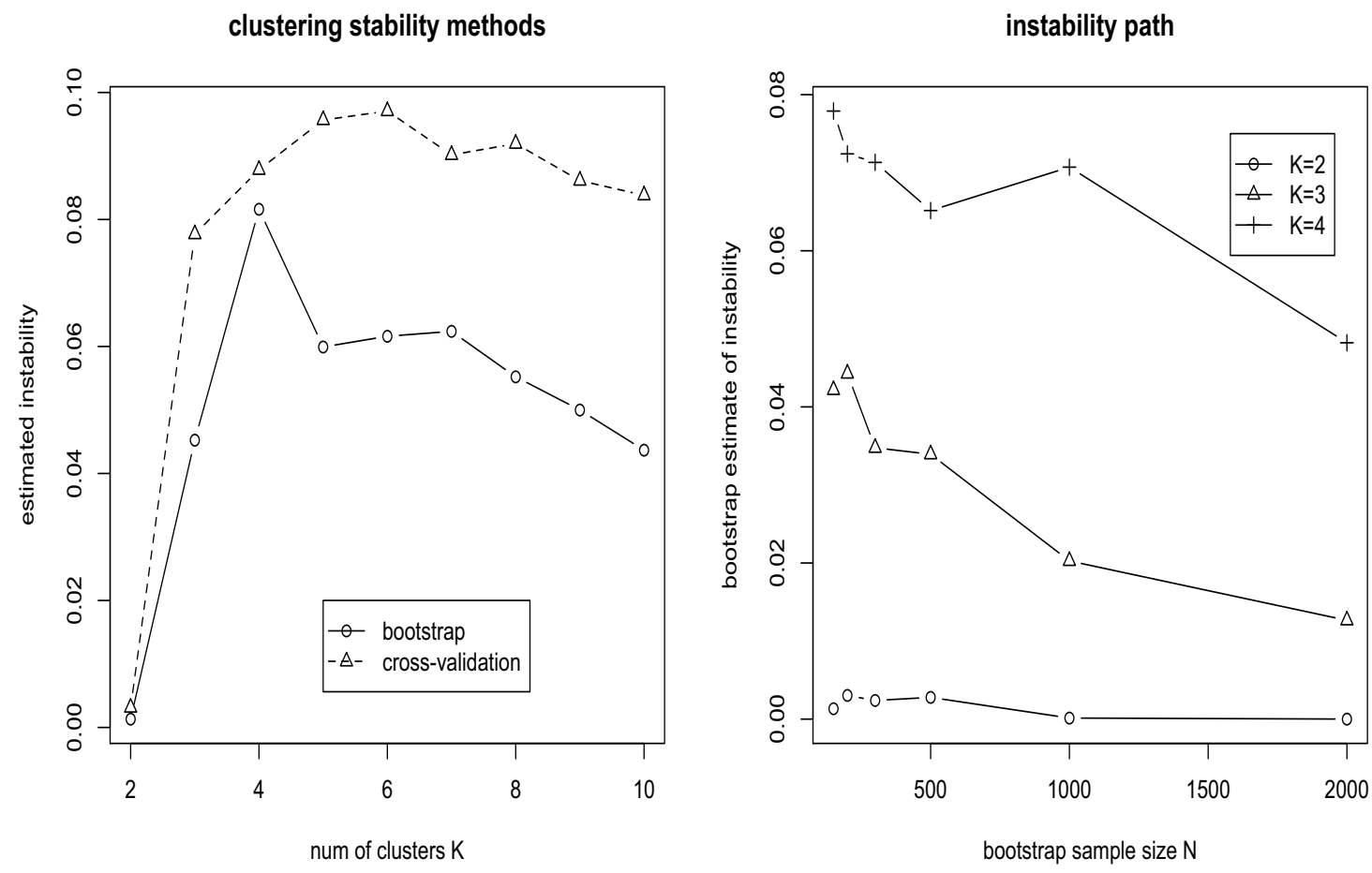
Figure 6: Wine data example $(\hat{k}=3)$
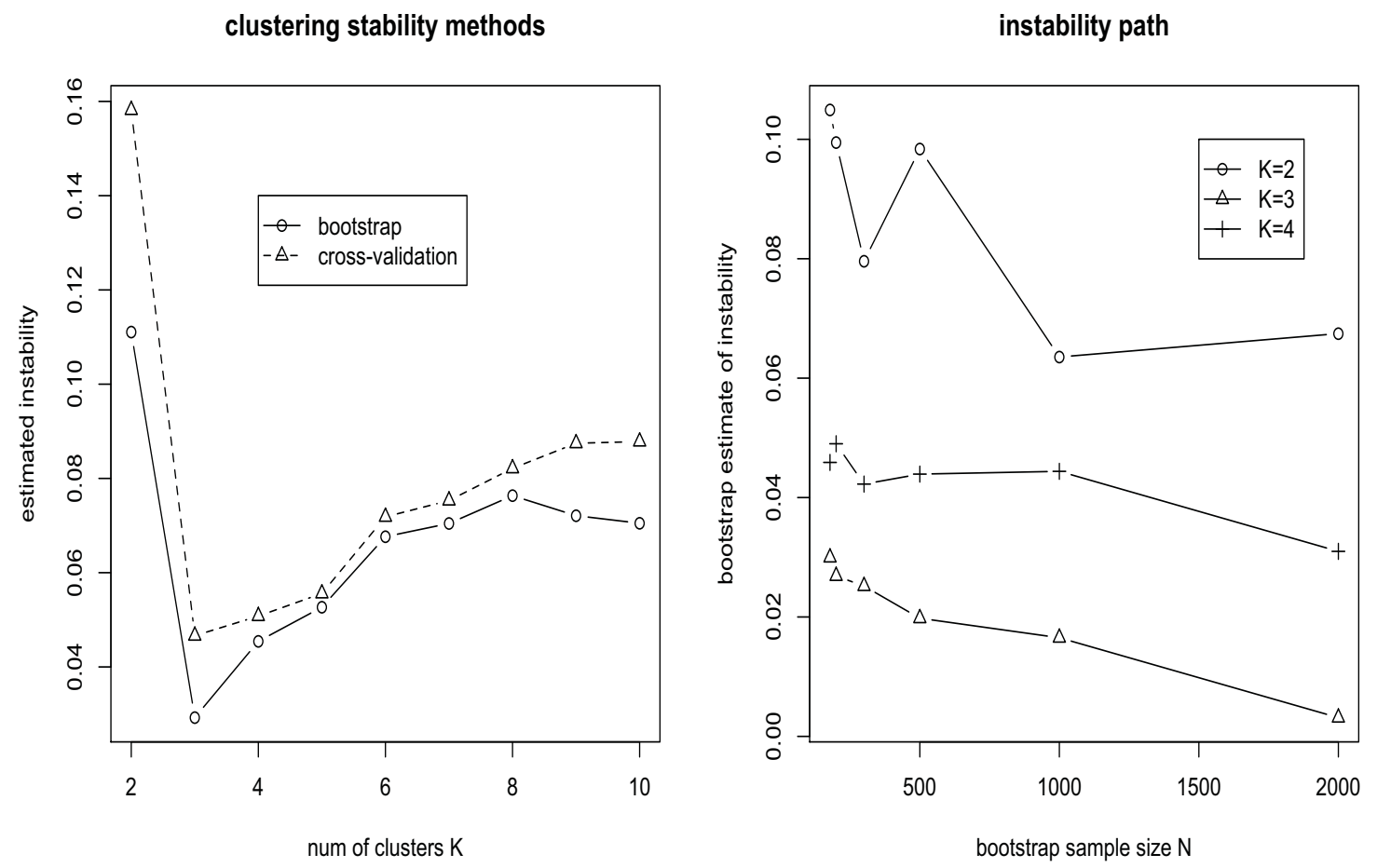